\title{
Accelerator driven system for transmutation and energy production
}

\author{
Petar Zhivkov ${ }^{1}$, Chavdar Stoyanov ${ }^{1}$ and Walter Furman ${ }^{2}$ \\ ${ }^{1}$ Institute for Nuclear Research and Nuclear Energy, Bulgarian Academy of Sciences, Sofia, Bulgaria \\ ${ }^{2}$ Joint Institute for Nuclear Research, Dubna, Russia
}

\begin{abstract}
The significant quantities of highly radiotoxic long-lived nuclear waste necessitate the research of methods of their transmutation to short-lived ones. This can be achieved by interaction between nuclear waste and neutrons (n,xn)-reactions and radiative captive). The ADS (Accelerator Driven System) is the most promising tool for such transmutation. ADS consists of powerful high energy accelerator irradiating a target of heavy nuclei: uranium, thorium, lead and bismuth. Inside of the target is generated a high-energy neutron field. Several ADS constructions for waste transmutation and energy production are considered. The influence of the cooling system on the neutron flux and the energy production in ADS are investigated. The radiation and biological protection are also discussed.
\end{abstract}

\section{Introduction}

Many experiments investigating the parameters and abilities of ADS (Accelerator Driven System) have been done in the last decade [1-6]. These investigations include computer simulations. Calculations of experiments with MCNPX, GEANT4, FLUKA transport codes are used. Most of the codes use group cross sections in contrast to MCNPX where precise nuclear data tables are included in the program. Most of the nuclear data tables are defined up to $20 \mathrm{MeV}$. Some of the tables are extended up to 100 or 150 , even up to $200 \mathrm{MeV}$. In the MCNP6 simulations ENDF60 cross sections for nat $\mathrm{Pb}$, ${ }^{235,238} \mathrm{U},{ }^{239,240} \mathrm{Pu}$ are used. All of them are defined up to $20 \mathrm{MeV}$ incident neutron energy. If there are no cross sections, the CEM, INCL, Bergini and evaporation ABLA models are used. The MCNP6 transport code is the most powerful code describing nuclear fission for different isotopes. There is one experiment where all particles deposit their energy in the volume of the experimental set up [7]. The experimental measurements of the neutron-induced fission and radiative captive are almost two times higher than the calculations. All calculations of neutron-induced reactions in ADS with significant amount of fission nucleus can be underestimated. Therefore, the experiments are very important for the validation of the nuclear models and cross sections.

\section{ADS components and their characteristics}

The ADS system consists of the following parts 1) Accelerator, 2) Target, 3) Reactor part and reflector, 4)
Radiation shielding of the reactor part, Fig.1, similar as in [8]. The present article does not discuss the accelerator. The aims of the article are investigation of energy production and equilibrium of the number of fission reactions and fuel production in the target, MOX fuel plus shielding. The energy can be produced by neutroninduced fission in isotopes ${ }^{233,235,238} \mathrm{U},{ }^{232} \mathrm{Th}$ and ${ }^{239,240} \mathrm{Pu}$. All calculations of neutron-induced reactions with energy up to $20 \mathrm{MeV}$ are made. The fissions induced by neutrons with energy $E_{n}>20 \mathrm{MeV}$ and charged particles are not included in this work. The design of ADS is simple because we are interested in integral parameters only such as total neutron production, leakage of neutrons and neutron-induced reactions. The ADS consists of 4 main parts. The first is an accelerator. A proton beam with energies from 1 to $2 \mathrm{GeV}$ is chosen. The second is a target zone made of ${ }^{235,238} \mathrm{U}$, nat $\mathrm{Pb},{ }^{239} \mathrm{Pu}$ and a combination of them. The third is a reflector and cooling system simultaneously made of nat $\mathrm{Pb}$. The fourth is nuclear fuel MOX (Mixed Oxide Fuel, 15-25\% concentration of isotopes ${ }^{239} \mathrm{Pu}+{ }^{235} \mathrm{U}$ ). For axial (direction of proton beam) symmetry the ADS has a cylindrical shape with approximate dimensions: diameter $\mathrm{D}=2.5 \mathrm{~m}$, length $\mathrm{L}=3$ $\mathrm{m}$ (see Fig. 1). The approximate masses of the nuclides without shielding ( ${ }^{\text {nat }} U$ and ${ }^{232} \mathrm{Th}$ ) are $\mathrm{M}_{\min }\left({ }^{\text {nat }} \mathrm{U}\right)=270 \mathrm{~kg}$, $\mathrm{M}_{\min }\left({ }^{239} \mathrm{Pu}\right)=109 \mathrm{~kg}, \mathrm{M}_{\min }\left({ }^{240} \mathrm{Pu}\right)=2 \mathrm{~kg}, \mathrm{M}_{\min }\left({ }^{\text {nat }} \mathrm{Pb}\right)=4.5$ $\mathrm{t}$. The masses of the ADS shielding are more than $100 \mathrm{t}$ and $60 \mathrm{t}$ of ${ }^{\text {nat }} \mathrm{U}$ and ${ }^{232} \mathrm{Th}$, respectively. Some changes in the size of ADS are made. It is necessary to analyse the stability and changes of $\mathrm{K}_{\text {eff }}$ and the integral number of neutron-induced reactions as a function of the size and nuclides in the target, MOX fuel and shielding (area of nuclear fuel production).

\footnotetext{
* Corresponding author: petar.zhivkov@gmail.com
} 


\subsection{Spallation target}

The neutrons in the target are generated by different processes. The number of neutrons depends on the nuclides included in the targets, the energy and type of the beam ions. Spallation neutrons and fragments from the targets can be produced by high-energy charged particles $1<\mathrm{E}<10 \mathrm{GeV} / \mathrm{n}$. The targets consist of heavy or/and fissionable nuclides such as ${ }^{n a t} \mathrm{~Pb}, \mathrm{~W}, \mathrm{Bi},{ }^{232} \mathrm{Th}$ and isotopes of uranium and plutonium. If the energy of incident neutrons is several hundreds $\mathrm{MeV}$, then the processes of fragmentation and pion creation are unlikely. The reaction $(\mathrm{n}, \mathrm{xn})$, high energy fission and neutron scattering will dominate. The main reactions for the isotopes of $\mathrm{Pb}$ and $\mathrm{Bi}$ are $(\mathrm{n}, \mathrm{xn})$, but $(\mathrm{n}, \mathrm{f})$ is also possible and the cross sections are mbarn. For fission isotopes such as $\mathrm{Th}, \mathrm{U}$ and $\mathrm{Pu}$ the main reactions are the following four: $(\mathrm{n}, \mathrm{xn})$, fission, radiative capture $(\mathrm{n}, \gamma)$ and neutron elastic/non-elastic scattering. The reactions $(n, \gamma)^{238} \mathrm{U}$, ${ }^{232} \mathrm{Th}$ after two beta decays can produce nuclear fuel ${ }^{239} \mathrm{Pu}$ and ${ }^{233} \mathrm{U}$. All the processes are illustrated below in (1) and (2). The reaction $(\mathrm{n}, \mathrm{f}){ }^{239} \mathrm{Pu}$ and $(\mathrm{n}, \mathrm{f})^{233} \mathrm{U}$ has cross sections of thousands of barns for thermal neutrons.

$$
\begin{gathered}
(\mathrm{n}, \gamma)^{238} \mathrm{U} \rightarrow{ }^{239} \mathrm{U} \stackrel{\beta-}{\rightarrow}{ }^{239} \mathrm{~Np} \stackrel{\beta-}{\rightarrow}{ }^{239} \mathrm{Pu} \\
(\mathrm{n}, \gamma)^{232} \mathrm{Th} \rightarrow{ }^{233} \mathrm{Th} \stackrel{\beta-}{\rightarrow}{ }^{233} \mathrm{~Pa} \stackrel{\beta-}{\rightarrow}{ }^{233} \mathrm{U}
\end{gathered}
$$

The cross sections of the reaction $(\mathrm{n}, \mathrm{f})^{235,238} \mathrm{U}$ and ${ }^{239,240} \mathrm{Pu}$ for neutrons with energy from 20 to $200 \mathrm{MeV}$ are in the interval $1-5$ bars and for ${ }^{232} \mathrm{Th}$ - less than 1 barn. The important characteristic is the multiplicity $\mu$ which is the number of the neutrons received per one fission [9]. For the isotopes ${ }^{235,238} \mathrm{U}$ and low energy of the incident neutrons $\mu$ is 2.4 neutrons per fission, but for neutrons with energy $E_{n}=200 \mathrm{MeV}$ the multiplicity $\mu$ is 12 and 13 , respectively [9]. For plutonium isotopes the multiplicity $\mu$ will be more than for ${ }^{238} \mathrm{U}$. Another important feature is the neutron fission spectrum and its mean energy $\left\langle\mathrm{E}_{\mathrm{n}}>\right.$. For thermal neutrons the mean energy of the fission neutrons is $\left\langle\mathrm{E}_{\mathrm{n}}\right\rangle=1.8 \mathrm{MeV}$, the spectrum has Watt distribution and the maximum energy on the fission neutrons is approximately $10 \mathrm{MeV}$.

The number of generated neutrons will be significantly higher if the isotopes ${ }^{233,235} \mathrm{U}$ and ${ }^{239} \mathrm{Pu}$ are included in the target. The target has significant energy load (fission and ionization) and the heat has to be transported to a cooler and exchanged. Lead and bismuth must be used as heating transporter in the targets. If fission nuclides like ${ }^{238} \mathrm{U}$ and ${ }^{232} \mathrm{Th}$ exist in the target, the production of ${ }^{239} \mathrm{Pu}$ and ${ }^{233} \mathrm{U}$ will start simultaneously with the fission and then $\mathrm{K}_{\mathrm{eff}}$ will increase. The targets have to be constructed in such a way so that the number of reactions (1) and (2) is less than the number of fissions, i.e. to reach an equilibrium between the number of fissions and the fuel production. ${ }^{\text {nat }} \mathrm{U},{ }^{\text {nat }} \mathrm{Pb}$ and ${ }^{239} \mathrm{Pu}$ are used in the simulations of the ADS target. The aim is to estimate the fissions and fuel production in the target. In case of nat $\mathrm{Pb}$ and $\mathrm{Bi}$ targets, there is no nuclear fuel production and their results are not shown here. The simulations use target materials ${ }^{\text {nat }} \mathrm{U},{ }^{238} \mathrm{U}$ with different concentration of ${ }^{239} \mathrm{Pu}$. The concentrations of ${ }^{239} \mathrm{Pu}$ do not exceed $7 \%, \mathrm{~K}_{\text {eff }}$ is approximately 0.99 and the Beam Power Gain reaches the maximum. The results of fission and fuel production in the target are shown in Table 1. For one type of target but different ${ }^{239} \mathrm{Pu}$ concentration in the MOX fuel there are significant differences in the number of neutron-induced reactions (Table 1). In that case the influence of MOX fuel on the spallation target is clear.

Table 1. Distribution of $(\mathrm{n}, \mathrm{f})$ and $(\mathrm{n}, \gamma)$ reactions in the target. The target consists of ${ }^{238} \mathrm{U}-24 \%,{ }^{239} \mathrm{Pu}-6 \%$ and ${ }^{\mathrm{nat}} \mathrm{Pb}-70 \%$. The energy of the proton beam is $\mathrm{E}_{\mathrm{p}}=2 \mathrm{GeV}$. The concentration of ${ }^{239} \mathrm{Pu}$ in MOX fuel is different, marked with $\mathrm{T} 1$ and $\mathrm{T} 2$.

\begin{tabular}{|c|c|c|c|c|}
\hline & \multicolumn{2}{|c|}{$(\mathrm{n}, \mathrm{f})$} & \multicolumn{2}{c|}{$(\mathrm{n}, \gamma)$} \\
\hline & ${ }^{238} \mathrm{U}$ & ${ }^{239} \mathrm{Pu}$ & ${ }^{238} \mathrm{U}$ & ${ }^{239} \mathrm{Pu}$ \\
\hline $\mathrm{T} 1$ & 17 & 118 & 56 & 21 \\
\hline $\mathrm{T} 2$ & 3 & 22 & 11 & 4 \\
\hline
\end{tabular}

The neutron field generated by nuclear fuel induces more fissions and capture reactions in the target. Calculations of the parameters and neutron-induced reactions in the ADS targets in free space are not recommended. Table 2 shows the neutron-induced reactions in ${ }^{\text {nat }} U$ target. The number of reaction $(n, \gamma)^{238} \mathrm{U}$ is 3 times more than the uranium fission, but for the combination of ${ }^{n a t} \mathrm{~Pb},{ }^{238} \mathrm{U}$ and ${ }^{239} \mathrm{Pu}$ we have more fissions than fuel production.

Table 2. Distribution of $(\mathrm{n}, \mathrm{f})$ and $(\mathrm{n}, \gamma)$ reactions in the target. The target T2 is nat $U$. The energy of the proton beam is $E_{p}=1$ $\mathrm{GeV}$.

\begin{tabular}{|c|c|c|c|}
\hline & \multicolumn{2}{|c|}{$(\mathrm{n}, \mathrm{f})$} & $(\mathrm{n}, \gamma)$ \\
\hline & ${ }^{238} \mathrm{U}$ & ${ }^{235} \mathrm{U}$ & ${ }^{238} \mathrm{U}$ \\
\hline $\mathrm{T} 2$ & 6.2 & 1 & 18 \\
\hline
\end{tabular}

Thus, the equilibrium point can be achieved. The calculations show that the number of fissions in the reactor part depends on the number of neutrons generated in the target. Calculations with nuclear models and cross sections up to $20 \mathrm{MeV}$ are done. The total number of generated neutrons can be more if the VassilkovGoldanski experiment is taken into account. The experimental estimations of [7] show that the total number of $(\mathrm{n}, \mathrm{f})$ reaction and $(\mathrm{n}, \gamma)$ are two times and 1.8 times more than calculated.

\subsection{Reflector part}

The aims of the reflector ${ }^{\text {nat }} \mathrm{Pb}$ are neutron scattering (reflection) and heating exchanger. It is a very important part because its construction determines the number of neutrons returned to MOX and increases the number of 
the fissions. Due to the fact that $85 \%$ of all fissions result from ${ }^{239} \mathrm{Pu}$ fission (which has thousands of barns for thermal neutrons) precise knowing of cross sections of low energy neutron reactions with nuclides ${ }^{206,207,208} \mathrm{~Pb}$ is crucial. MCNP6 code allows to use different cross section libraries. For lead isotopes ENDF60, ENDF70, LA150, TENDL and others are available. They are defined up 20 to $200 \mathrm{MeV}$. For more reliable results of neutron-induced reactions in ADS simulations, the influence of different lead cross section data should be investigated. It is not within the scope of this work and only ENDF60 is used.

\subsection{Reactor part}

It consists of MOX (Mix-Oxide full) with ${ }^{239} \mathrm{Pu}$ and ${ }^{235} \mathrm{U}$ concentration of approximately $20 \%$. The cooling system is not included, but it is supposed to be gas, pipes located in the reactor and reflector part. The $\mathrm{K}_{\text {eff }}$ of ADS quantity of MOX fuel, reflector or target materials. The neutroninduced fission and capture reactions are calculated. More than $90 \%$ of all fissions come from reaction $(\mathrm{n}, \mathrm{f})^{239} \mathrm{Pu}$, $\mathrm{E}_{\mathrm{n}}<20 \mathrm{MeV}$ which has very high cross sections for low energy neutrons. The $(\mathrm{n}, \mathrm{f})^{239} \mathrm{Pu}$ reaction dominate in all types of fissions and it is $85 \%$ approximately. The Beam Power Gain (BPG) coefficient is defined as a ratio of the total energy production and the beam energy i.e. $\mathrm{BPG}=\left(\mathrm{E}_{\text {fiss }}+\mathrm{E}_{\text {ion }}\right) / \mathrm{E}_{\mathrm{p}}$ or $\mathrm{BPG}_{1}=\left(\mathrm{E}_{\text {fiss }}+\mathrm{E}_{\text {ion }}+\mathrm{E}_{(\mathrm{n}, \gamma)}\right) / \mathrm{E}_{\mathrm{p}}$. To better estimate the possible energy gain from ADS, the nuclear fuel production in reactions (1) and (2) are added

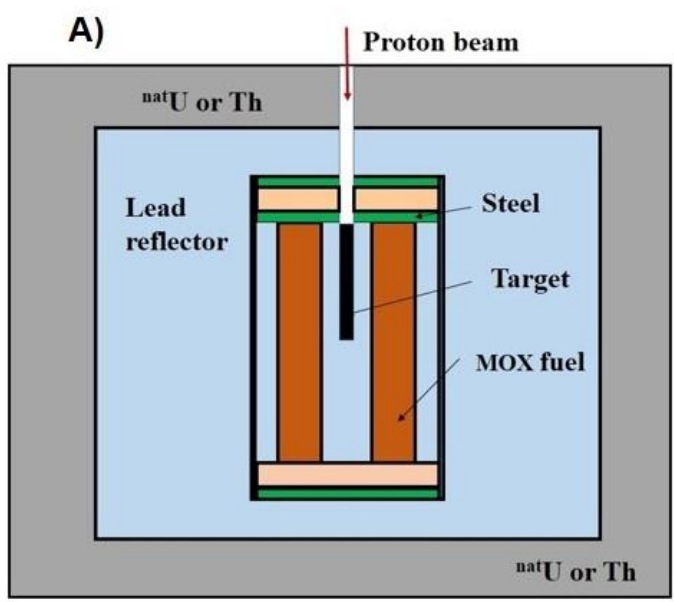

Figure 1. Cross sections of ADS. A) - axial cross section of ADS ; B) - cross section perpendicular to the axis of cylinder. Target zone, MOX, reflector ${ }^{\text {nat }} \mathrm{Pb}$, and ${ }^{\text {nat }} \mathrm{U}$ or $\mathrm{Th}$ protection shield. $\mathrm{K}_{\mathrm{eff}}=0.98-0.99$. The size is approximately $2.5 \times 3 \mathrm{~m}$.

into BGP. The nuclear fuel can be produced in the target, MOX fuel and shielding made by uranium and thorium232. The number of radiative capture (nuclear fuel production) must be significantly lower in the reactor part than the number of fissions. This is required due to the fact that the ADS safety must be priority. The equilibrium in the reactor part must not be achieved. For the simulations of neutron-induced reactions with ${ }^{235,238} \mathrm{U}$, ${ }^{239,240} \mathrm{Pu}$ and ${ }^{232} \mathrm{Th}$ the ENDF60 nuclear data tables are used. They are defined for neutrons with incident neutron energy up to $20 \mathrm{MeV}$. For the simulations two proton beams $\mathrm{E}_{\mathrm{p}}=1$ and $2 \mathrm{GeV}$ are used, Table 3. Some of the results are shown in Table 2 . In Case 1 the proton beam has energy $E_{p}=1 \mathrm{GeV}$ and ${ }^{\text {nat }} \mathrm{U}$ target, Table 3 . In Case 2 the target consists of $6 \%{ }^{239} \mathrm{Pu} 24 \%{ }^{\text {nat }} \mathrm{U}$ and ${ }^{\text {nat }} \mathrm{Pb}$, Table 3 . In both cases the protection shield is made of ${ }^{232} \mathrm{Th}$. The Keff for case 2 is higher and is approximately 0.99 . The closer $\mathrm{K}_{\text {eff }}$ to 1 , the higher the number of fissions and capture reactions in ADS. Very small variations of the ${ }^{239} \mathrm{Pu}$ concentration in the target and in the MOX fuel or variations in the reflector size or other geometrical parameters change the total fission and capture reactions rapidly. This results in variation of BPG coefficient. Stable work of ADS with significant power production can be achieved.

Table 3. Distribution of $(n, f)$ and $(n, \gamma)$ reactions in the ADS. The target is nat $U$. The energy of the proton beams is $E_{p}=1$ for Case 1 and $\mathrm{E}_{\mathrm{p}}=2 \mathrm{GeV}$ for Case 2 .

\begin{tabular}{|l|l|l|l|l|}
\hline & \multicolumn{2}{|l|}{ MOX+target } & Shield & BPG \\
\hline & $(\mathrm{n}, \mathrm{f})$ & $(\mathrm{n}, \gamma)$ & $(\mathrm{n}, \gamma)$ & \\
\hline Case 1 & 1150 & 310 & 1220 & 230 \\
\hline Case 2 & 9500 & 2600 & 11000 & 940 \\
\hline
\end{tabular}

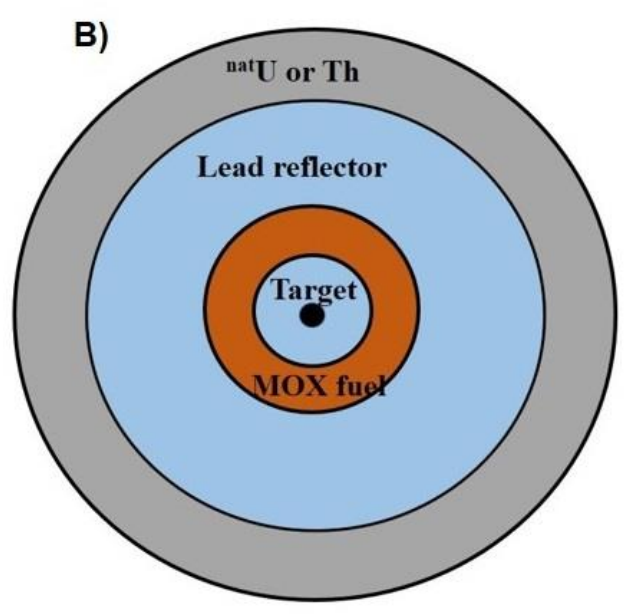

\subsection{Shielding or nuclear fuel production area}

The shield aims to protect humans and the environment from ADS radiation. The neutron leakage is significant and neutrons always interact with nucleus. Due to the low energy the neutrons participate in radiative captive and there is matter activation. Neutron leakage means energy losses and reduced ADS efficiency. The most effective way to construct radiation protection shield and increase the ADS efficiency is to use neutrons for nuclear fuel production, see reaction chains (1) and (2). In the simulations ${ }^{232} \mathrm{Th}$ and ${ }^{\text {nat }} U$ are used as shielding. The cross sections for $(\mathrm{n}, \mathrm{f})^{232} \mathrm{Th} \mathrm{E}_{\mathrm{n}}<1 \mathrm{MeV}$ are very low and this 
reaction does not occur, only radiative captive can be observed. In case of ${ }^{\text {nat }} U$ which has $0.7 \%$ concentration of ${ }^{235} \mathrm{U}$ (high fission cross section for thermal neutron) the situation is different. There is fission of nat $U$ in the shielding and activation is expected. This applies to ${ }^{232} \mathrm{Th}$ also, because ${ }^{233} \mathrm{U}$ is accumulated during the irradiation of ADS. The problem can be solved if the shielding is in a liquid form and ${ }^{233} \mathrm{U}$ and ${ }^{239} \mathrm{Pu}$ are removed constantly.

\section{Discussion}

The stable work of ADS can be done by proton beam (energy and current), concentration of fissionable nuclides in the target and MOX fuel and ${ }^{\text {nat }} \mathrm{Pb}$ reflector. Proton beam parameters can be changed immediately while the other are part of the ADS construction. The target must generate constant neutron field. It can be archive by equilibrium of fissions and nuclear fuel production. Such equilibrium must not be achieved in reactor part. It necessary for nuclear security for stable work of ADS. In that case the significant neutron leakage will be generated and the efficiency of the ADS will be decrees. Thus the activation of construction material and significant energy losses. The solution of the problem can be found in construction of ${ }^{232} \mathrm{Th}$ and ${ }^{\mathrm{depl}} \mathrm{U}$ protection shield for nuclear fuel production. Very important part of such ADS is reflector. The reflector moderate neutrons with low energy. All cross sections for low energy induced reactions in MOX and reflector are important. Another significant sources of errors are reconstruction of high energy component of spallation neutrons and high energy fission in the target region. The high energy fission is describe by nuclear models, not from cross sections. The multiplicity and neutron fission spectrum are very important [9]. The verification of high energy nuclear modes with experimental data received from irradiation of massive ${ }^{\text {depl }} U$ target can be received from future experiments in JINR, Dubna [10]. The experimental set up is uranium cylinder with 21 tons packed with steel case. These experiments can verify not only nuclear models but Vassilkov-Goldanski experiment also [7].

\section{Conclusion}

The ADS has potential for production of energy from heavier than uranium nuclides, which are unacceptable for reactor based on thermal neutrons. The equilibrium of fission and capture reactions in the target can be reached by different concentration of uranium and plutonium isotopes. The number of fissions in the reactor part have to be three times more than the number of capture reactions. It is necessary for nuclear security. The Beam Power Gain (BPG) vary in interval $10^{2}$ up to $10^{3}$ or more. It is very promising result for the energy production. The most important influence for fission reactions are the concentration of ${ }^{239} \mathrm{Pu},{ }^{235,238} \mathrm{U}$ and reflector, which modify the neutron spectra.

\section{References}

[1] S. Andriamonje, A. Angelopoulos, A. Apostolakis, et al., Phys. Lett. B 348, 697-709 (1995)

[2] http://sckcen.be/en/Technology future/MYRRHA

[3] https://ntof-exp.web.cern.ch/ntof-exp/

[4] M. Krivopustov, A. Pavliouk, A. Kovalenko, I. Mariin et al., J. Rad. Nucl. Chem. 279, n. 2, 567584 (2009)

[5] W. Furman, J. Adam, A. Baldin, et al., E\&Tr collaboration, PoS Baldin-ISHEPP-XXI (2012) 086

[6] A. Polański, P. Zhivkov, Ch. Stoynov, NATO Security through Science Series C: Environmental Security, 333-341 (2009)

[7] R.Vassilkov, V. Goldansky, B. Pimenov and Yu. Pokotilovsky, Atomic Energy 44, 329 (1978)

[8] P. Zhivkov, J. Phys.: Conf. Series 1023(1), 012031 (2018)

[9] Th. Ethvignot, M. Devlin, Th. Granier, R. Haigh, R. Nelson, J. O'Donnell and D. Rochman, AIP Conf. Proceedings 769(1), 656 (2005)

[10] P. Zhivkov, W. Furman, Ch. Stoyanov, J. Phys.: Conf. Series 533, 012053 (2014) 